\title{
Modafinil for the Treatment of Methamphetamine Dependence
}

\author{
Ann L. Anderson ${ }^{1}$, Shou-Hua Li ${ }^{1}$, Kousick Biswas ${ }^{2}$, Frances McSherry ${ }^{2}$, Tyson Holmes ${ }^{3}$, \\ Erin Iturriaga ${ }^{1}$, Roberta Kahn ${ }^{1}$, Nora Chiang ${ }^{1}$, Thomas Beresford ${ }^{4}$, Jan Campbell ${ }^{5}$, William \\ Haning ${ }^{6}$, Joseph Mawhinney ${ }^{7}$, Michael McCann ${ }^{8}$, Richard Rawson ${ }^{9}$, Christopher Stock $^{10}$, \\ Dennis Weis ${ }^{11}$, Elmer $\mathbf{Y u}^{12}$, and Ahmed M. Elkashef ${ }^{1}$ \\ ${ }^{1}$ Division of Pharmacotherapies and Medical Consequences of Drug Abuse, National Institute on \\ Drug Abuse, National Institutes of Health, Bethesda, MD \\ ${ }^{2}$ Coop Studies Program, VA Maryland Healthcare Center, Perry Point, MD \\ ${ }^{3}$ Department of Psychiatry and Behavioral Sciences, Stanford University School of Medicine, \\ Stanford, CA \\ ${ }^{4}$ Psychiatry Service, VA Medical Center, Denver, CO \\ ${ }^{5}$ University of Kansas Medical Center, Kansas City, KS \\ ${ }^{6}$ John A. Burns School of Med, Univ. of Hawaii, Honolulu, HI \\ ${ }^{7}$ South Bay Treatment Center, San Diego, CA \\ ${ }^{8}$ Matrix Inst. on Addictions, West Los Angeles, CA \\ ${ }^{9}$ UCLA Integrated Substance Abuse Program, Los Angeles, CA \\ ${ }^{10}$ VA Salt Lake City Healthcare System, Salt Lake City, Utah \\ ${ }^{11}$ lowa Lutheran Hosp., Des Moines, IA \\ ${ }^{12}$ Philadelphia VA Medical Center, Philadelphia, PA
}

Abstract

Aim-Modafinil was tested for efficacy in decreasing use in methamphetamine-dependent participants, compared to placebo.

Methods-This was a randomized, double-blind, placebo-controlled study, with 12 weeks of treatment and a 4-week follow-up. Eight outpatient substance abuse treatment clinics participated

Corresponding Author: Ann L. Anderson, DPMC - National Institute on Drug Abuse - NIH, 6001 Executive Blvd, Rm-4149, Bethesda, MD 20892-9551, Phone: (301) 435-0767, Fax: (301) 443-2599, aa135m@nih.gov.

Contributors: Authors Ann Anderson and Ahmed Elkashef wrote the first draft of the manuscript, and Ann Anderson edited the final draft. Shou-Hua Li, Kousick Biswas, Frances McSherry and Tyson Holmes did the statistical design, analyses, reports and editing. Erin Iturriaga contributed literature searches and descriptions of the study population and treatments. Roberta Kahn summarized the adverse events data. Nora Chiang provided chemical assay materials. Elmer Yu and Ahmed Elkashef contributed summaries of previous related work. Thomas Beresford, Jan Campbell, William Haning, Joseph Mawhinney, Michael McCann, Richard Rawson, Christopher Stock, and Dennis Weis were site Principle Investigators, and edited both the protocol and the manuscript. All authors have approved the final manuscript.

Conflict of Interest: All authors declare that they have no conflicts of interest, i.e., no actual or potential conflict of interest including any financial, personal or other relationships with people or organizations within three (3) years of beginning the work submitted that could inappropriately influence, or be perceived to influence, this work.

Publisher's Disclaimer: This is a PDF file of an unedited manuscript that has been accepted for publication. As a service to our customers we are providing this early version of the manuscript. The manuscript will undergo copyediting, typesetting, and review of the resulting proof before it is published in its final citable form. Please note that during the production process errors may be discovered which could affect the content, and all legal disclaimers that apply to the journal pertain. 
in the study. There were 210 treatment-seekers randomized, who all had a DSM-IV diagnosis of methamphetamine dependence; 68 participants to placebo, 72 to modafinil $200 \mathrm{mg}$, and 70 to modafinil 400mg, taken once daily on awakening. Participants came to the clinic three times per week for assessments, urine drug screens, and group psychotherapy. The primary outcome measure was a methamphetamine non-use week, which required all the week's qualitative urine drug screens to be negative for methamphetamine.

Results-Regression analysis showed no significant difference between either modafinil group (200 or 400mg) and placebo in change in weekly percentage having a methamphetamine non-use week over the 12-week treatment period $(\mathrm{p}=0.53)$. Similarly, a number of secondary outcomes did not show significant effects of modafinil. However, an ad-hoc analysis of medication compliance, by urinalysis for modafinil and its metabolite, did find a significant difference in maximum duration of abstinence ( 23 days vs. 10 days, $\mathrm{p}=0.003$ ), between those having the top quartile of compliance ( $>85 \%$ urines modafinil,$+ \mathrm{N}=36$ ), and the lower three quartiles of modafinil 200 and $400 \mathrm{mg}$ groups $(\mathrm{N}=106)$.

Conclusions-Although these data suggest that modafinil, plus group behavioral therapy, was not effective for decreasing methamphetamine use, the study is probably inconclusive because of inadequate compliance with taking medication.

\section{Keywords}

Methamphetamine; Substance-Related Disorders; drug therapy [subheading]; Modafinil; Medication Adherence; Attention Deficit Hyperactivity Disorders

\section{Introduction}

Methamphetamine (meth) is a synthetic stimulant which, along with other amphetamines, was widely prescribed in the US in the 1950's and 60's for obesity and 'depression' (Grinspoon and Hedblom, 1975). The drug's label was revised by the Food and Drug Administration (FDA) in 1975 to that of a Class II controlled substance. Although still approved for Attention Deficit Hyperactivity Disorder (ADHD) in children older than six, and as a short-term adjunct for obesity, the drug is often manufactured illegally in small 'home' or large 'super' labs, using ephedrine, pseudoephedrine, or P-2-P (phenyl-2propanone) as a precursor (Drug Enforcement Administration, 2005). In 2009, about 1.2 million Americans age 12 and older had used methamphetamine illicitly at least once in the year prior to being surveyed (Substance Abuse and Mental Health Services Administration, 2010). The United Nations estimated that there were about 14-53 million annual users globally, among persons aged 15 to 64 years (UNODC, 2010).

Dependence on methamphetamine can be a devastating illness, with ill effects and associations across the lifespan, at home and in the community. Neonates who were prenatally exposed to meth had a higher incidence of being small for gestational age, after adjusting for alcohol and tobacco exposure and other covariates (Nguyen D, et al, 2010). A sample of 526 meth dependent adults, who took part in the Methamphetamine Treatment Project of SAMHSA's Center for Substance Abuse Treatment, were reassessed three years later. Almost half of them (48\%) met DSM-IV criteria for a current or past psychiatric disorder other than substance use, and 29\% had antisocial personality disorder (GlasnerEdwards et al., 2010). Three of the major illicit drugs associated with criminality were assessed over a10 year follow-up. The courses of almost 1800 heroin, cocaine, and methamphetamine users showed that meth users had earlier initiation of drug use than cocaine users (age 19.6 vs $23.0 \mathrm{yrs}$ ), and spent more months incarcerated than cocaine users (10.5 vs. 6.3), though less than heroin users (34.7). Meth users were also more likely to be 
women than were heroin or cocaine users ( $46 \%$ vs. $11 \%$ or $29 \%$ ), and had a relatively high percentage of drug injection (44\% vs. $90 \%$ or $27 \%$; Hser et al., 2008).

In contrast to methamphetamine, modafinil is a novel, non-amphetamine psychostimulant, first approved by the US Food and Drug Administration (FDA) in 1998 for the treatment of narcolepsy, and later for shift work sleep disorder, and as an adjunct for obstructive sleep apnea (Cephalon, Inc., 2007). It is an effective wake-promoting agent, but its mechanism of action differs in some ways from that of the amphetamines, cocaine, or methylphenidate (Ballon and Feifel, 2006). Modafinil has distinctive actions on the hypocretin / orexin system (Scammell et al., 2000) and the glutamate / GABA (gamma-Aminobutyric Acid) systems (Ferraro et al., 1999), as well as some dopamine-mediated (Wisor et al., 2001) and alpha-adrenergic effects (Stone et al., 2002) that resemble those of more traditional stimulants.

There are several rationales for using modafinil to treat methamphetamine dependence. For example, modafinil has stimulant properties, which could be therapeutic for alleviating some stimulant withdrawal symptoms (McGregor et al., 2008). In animal testing it has been found to attenuate reinstatement of meth self-administration (Reichel and See, 2010). Also, modafinil appears to have lower abuse potential than methylphenidate or amphetamine (Myrick et al., 2004). Modafinil improves cognition and mood (Turner et al., 2004; Taneja et al., 2007), and has shown efficacy in the treatment of child and adult ADHD (Lindsay et al., 2006). Modafinil has been used in several trials of treatment for cocaine dependence, with mixed results (Dackis et al., 2005; Anderson et al., 2009). Finally, modafinil was safe and well tolerated in a Phase I interaction study with intravenous methamphetamine (unpublished report, Jones, 2007).

\section{Method}

The objectives of this study were to evaluate the efficacy and safety of modafinil relative to placebo, for reducing methamphetamine use in meth-dependent outpatients, as assessed by qualitative or quantitative urine drug screens. The study had a double-blind, placebocontrolled, parallel-group design in which, after a 3-week screening/baseline period, participants were randomly assigned with equal probability to one of three treatment groups to receive 400mg modafinil, 200mg modafinil, or matched placebo daily for 12 weeks, with a follow-up assessment 4 weeks after treatment completion. Adaptive "urn" randomization (Wei and Lachin, 1988) was used to balance treatment groups within site, based on gender, current ADHD, and self-report of the frequency of methamphetamine use in the last 30 days before consent $(\leq 18$ days versus $>18$ days).

\subsection{Study Population}

Eight outpatient drug treatment clinic sites received IRB approval to recruit about 26 participants each, using print and radio ads, and to pay participants for coming to clinic three times weekly to provide a urine sample and other research data. A total of 210 participants who met criteria for methamphetamine dependence, determined by the Structured Clinical Interview (SCID) of the Diagnostic and Statistical Manual of Mental Disorders Fourth Edition (DSM-IV), were randomized into the three treatment groups ( $\sim 70$ participants per group). Participants with the ability to understand and provide written informed consent were at least 18 years old, and provided at least one meth-positive urine within the 3-week screening/baseline period prior to randomization. Urines were tested three times per week, and inclusion required at least 4 baseline urines. Exclusion criteria included: dependence on a drug other than methamphetamine, marijuana or nicotine, dependence on alcohol current or past, or physiological dependence on a sedative-hypnotic (e.g., a benzodiazepine) requiring medical detoxification. Some other exclusions were: pregnancy or lactation, taking 
a medication that could interact adversely with modafinil, serious medical illness including AIDS and Stage I hypertension, psychiatric disorder requiring medication treatment, and court-mandated urine toxicology test results as part of treatment for methamphetamine dependence.

\subsection{Treatments}

During the 12 weeks of outpatient treatment, participants took a 200 or $400 \mathrm{mg}$ tablet of modafinil, or the matched placebo, once daily on awakening. All participants received standardized psychosocial therapy, which consisted of Matrix Institute (http://www.matrixinstitute.org/) manual-guided Cognitive Behavioral Therapy, 90-minute group sessions, three times per week during the 12-week treatment period. All were offered (referral for) Human Immunodeficiency Virus (HIV) counseling and testing. One session of Motivational Enhancement Therapy was provided in the third week of screening/baseline visits.

\subsection{Safety Assessments}

Candidates for study enrollment had a medical history and physical examination, a 12-lead electrocardiogram (ECG), clinical laboratory studies (blood chemistry, hematology, urinalysis, and pregnancy test if female), and Hamilton Depression Rating Scale (HAM-D) during screening or baseline. Vital signs, concomitant medication use, and a urine screen for other substances of abuse were assessed weekly. Repeat HAM-D and pregnancy tests were assessed at Weeks 4, 8, and 12. Adverse Events (AEs) were assessed at each study visit and reviewed weekly by the site physician. At Treatment-Week-12 or study discontinuation, all participants had a repeat physical examination, clinical laboratory studies, and ECG.

\subsection{Efficacy Assessments}

Severity of methamphetamine dependence was evaluated by comparing groups on the average change in scores of the Addiction Severity Index (ASI), Brief Substance Craving Scale (BSCS), and Clinical Global Impression, as assessed by participant's self-report (CGI$\mathrm{S})$ and observer report (CGI-O). The ASI was measured at baseline, and the ASI-Lite followed up at Weeks 6 and 12. The BSCS, CGI-S, and CGI-O were obtained weekly during the baseline and treatment periods. The HIV Risk-Taking Behavior Scale (HRBS) was assessed at baseline and at Week-12 or the last study visit.

The presence of current Attention Deficit / Hyperactivity Disorder was assessed in screening by the Adult ADHD Clinical Diagnostic Scale (ACDS) V 1.2, a semi-structured interview to document current symptoms for the diagnosis of ADHD in an adult. The Adult ADHD Investigator Symptom Rating Scale (AISRS) uses the same 18 items and probes (of ADHD domains in the DSM-IV) to take repeated measures of the symptoms and observe changes over time. It is meant to improve inter-rater reliability (Adler et al., 2005), and was administered at baseline and Weeks 4, 8, and 12. In addition, we used the 30-item Barratt Impulsiveness Scale at baseline and Weeks 6 and 12. To test executive functioning and other cognitive abilities for a response to modafinil, we used 6 tests from the Cambridge Neuropsychological Test Automated Battery $\left(\mathrm{CANTAB}^{\circledR}\right)$, a series of cognitive tests administered on a touch screen tablet PC, assessed at screening, baseline, Week 6 and Week 12. The data for these tests were analyzed by Cambridge Cognition, Ltd. (Cambridge, UK).

\subsection{Analysis Plan}

It was hypothesized that modafinil, compared to placebo, would increase the number of methamphetamine non-use weeks (a week in which all of the qualitative urine drug screens within the week were negative for meth), over the treatment period. Some secondary 
outcomes included treatment group comparisons of the maximum number of consecutive non-use days, and of the proportion of 'successful' participants (e.g., who achieved 21 consecutive days of abstinence any time in the trial, or who decreased self-report meth-usedays to $50 \%$ of baseline).

Each primary and secondary outcome variable was analyzed using the intent-to-treat population, defined as the participants who were randomized to treatment and received the first dose of study agent. The outcomes that had repeated measures were analyzed using Generalized Estimating Equations (GEE), adjusted for factors of treatment group (three levels of medication dose: 0, 200 and 400mg), linear study week (1-12), and the interaction between group and week. Various models were tested, including with covariates for gender, current ADHD, or level of methamphetamine use during the 30 days prior to consent $(\leq 18$ days out of 30 vs. >18d). Statistical tests were two-sided at a 5\% Type I error rate.

Treatment compliance (medication or counseling) was evaluated based on the percent of days medication was taken or counseling sessions were attended $(3 \times / w k)$, out of a) the total scheduled for 12 weeks, and $b$ ) the total scheduled up to the point that the participant left the study. For the medication compliance assessment, self-report of use was aided by pill count from any returned dispensing cards. Study retention was calculated based on each participant's number of days from randomization to last study visit. A 'survival' curve shows the percent of participants remaining in the study, each day over the possible total treatment length of 84 days. (After six consecutive missed visits, participants were discontinued from the study.)

For evaluation of medication treatment effect on methamphetamine abuse, the primary outcome analysis used GEE to compare treatment groups' change in methamphetamine nonuse weeks. The "non-use weeks" outcome has been used in our previous meth studies and employs only urine toxicology, which is more accurate than self-report although there is also more missing data. GEE is a model-based regression method, appropriate for the analysis of correlated data that result from repeated measures in a longitudinal study. It assumes that missing data is missing completely at random. For this analysis we used weekly intervals, instead of the 36 variably-spaced possible urine samples, in order to decrease the complexity of the correlation structure. The SAS GENMOD procedure (SAS Institute Inc., Cary, North Carolina) was used to estimate and test the models.

We also analyzed an outcome for efficacy that demonstrates 'terminal abstinence.' This outcome compares the proportion of participants in each Group who have 'success' at the end of the trial, i.e., participants demonstrate abstinence in the last two weeks of the study (Weeks 11 and 12) by having all qualitative urine screens negative, providing at least two urines for each week. Participants whose urine was missing for any longer than three days of the last 2 weeks, were counted as 'failure.' For this outcome, only participants who remain until the end of the trial are included in the results.

Other secondary outcomes included the following:

1. for quantitative methamphetamine use, the decrease in average weekly $\log _{10}$ median urine methamphetamine concentration over the 12-week treatment period;

2. any of seven Addiction Severity Index (ASI)-Lite domains, examining the difference in mean change scores from baseline to last observation;

3. either of the two outcomes (improvement or severity) from the Clinical Global Impression scales, using GEE, separately regressed on treatment Group and the Group $\times$ Week interaction, for the Observer or for the Self; 
4. the change in BSCS total score (range 0 to 12), analyzed using GEE over the treatment period;

5. the HIV Risk Behavior Scale (HRBS), containing a sexual risk domain and a drug injection domain, change scores from baseline to Week 12/13.

6. the Adult ADHD Investigator Symptom Rating Scale change scores, from baseline to either Week 4, Week 8, Week 12, or the last available score.

7. the proportion of participants who improved (decreased their score) by a threshold of $30 \%$ or more on the AISRS.

8. the Barratt's Impulsiveness Scale, ANOVA comparisons of scores at baseline, Week 6 or Week 12, using the total scale, or the subscales for Attention, Motor, or Non-planning Impulsivity. We also divided the total sample into 'normal' vs. 'high' impulsivity groups (<72 vs. $\geq 72$ score, BIS) and analyzed with GEE.

Analyses of the CANTAB cognitive tests included ANOVA to compare modafinil $200 \mathrm{mg}$ to placebo and modafinil 400mg to placebo, using change from baseline measures to Week 6 , Week 12, and 'last available score.' Where data were not normally distributed even after transformations, analyses were non-parametric using Wilcoxon Rank Sum test. All statistical tests were performed 2-sided at a 5\% Type 1 error rate, and with Dunnett's multiple comparison test.

Baseline characteristics of the participants in each treatment group were summarized to demonstrate the results of randomization. Chi-square or Fisher's Exact tests were used for comparisons. A summary was prepared of dropouts, i.e., study retention over time, by treatment group, and for a priori defined subgroups. The number of missing observations was compared between treatment groups, and for a priori defined subgroups. All AEs were MedDRA-coded and reported in tabular form, indicating the frequency of each 'preferred term' type of AE by treatment group. AE's leading to discontinuation of study medication were also tabulated.

\section{Results}

\subsection{Baseline Demographics}

Figure 1 is a flow diagram of the numbers of study participants, from consent through randomization and on to completion of the study. The screen failure rate was $51 \%$ (226/440). The most common reasons for failure in screening were: 1] not willing and able to comply with study procedures, including 'did not return to clinic' (54\%); 2] did not provide a methamphetamine-positive urine during the 3-week screening period (19\%); 3] had current dependence on a psychoactive substance other than meth, nicotine or marijuana, or required sedative detox, or had current or past alcohol dependence $(11 \%)$; 4] was taking a medication that could interact adversely with modafinil $(6 \%) ; 5]$ had a serious medical illness such as uncontrolled hypertension or diabetes, or significant cardiovascular, hepatic, renal disease, etc., (6\%); and 6] had a psychiatric disorder requiring ongoing medication $(5 \%)$.

Demographics and baseline characteristics of study participants are summarized in Table 1. We detected no significant differences between treatment groups on these baseline characteristics.

\subsection{Treatment Retention and Medication Compliance}

A total of 112 participants (53\%) completed the 12 weeks of treatment, with no significant differences in retention between groups ( $\mathrm{p}=0.91$, log-rank test, Figure 2$)$. Retention also did 
not differ significantly between those who were low-to-moderate vs. heavy users ( $\mathrm{p}=0.96$, $\log$-rank test), or by gender ( $\mathrm{p}=0.15)$ or ADHD status ( $\mathrm{p}=0.44)$. Medication compliance by self-report (aided by pill count) was similar across all treatment groups, averaging $93 \%$ (range $14-100 \%$ ) of the expected number of tablets taken, up until the individual's point of study termination.

An ad-hoc analysis of medication compliance, using the first urine specimen of each week to analyze for modafinil and its metabolite, found a consistently lower compliance percentage than that reported by pill count. Our conventional assessment of medication compliance, self-report augmented by pill count, showed only weak agreement with the observed urine measure (Intraclass correlation coefficient $=0.21$, Fig 3). Among the 142 participants who took modafinil, compliance in the top quartile showed $>85 \%$ of weekly urines were positive for a measurable amount of modafinil (data not shown). The second quartile had $75-83 \%$ of urines positive. The third quartile had $42-67 \%$ positive, and the fourth quartile had $\leq 40 \%$ of urines positive, including 14 individuals (10\%) who had 0 urines positive for modafinil.

\subsection{Primary Outcome - Efficacy by Urine Analysis}

Our simple GEE model contained only factors for treatment Group, Study Week, and their interaction, Week $\times$ Group (which represented the medication effect). The primary outcome was methamphetamine non-use weeks, based on urine toxicology. This model showed no significant interaction between treatment Groups (overall) and Study Week in the rate of change (slope) in the primary outcome over the 12-week treatment period (see Fig. 4, $\mathrm{p}=0.53$, GEE). In the simple model the factor of Study Week alone, or time in treatment, did have a significant effect $(\mathrm{p}<0.001)$, while treatment Group did not $(\mathrm{p}=0.50)$.

We also tested a secondary outcome 'maximum number of consecutive methamphetamine non-use days' (by qualitative urine screens), and found no significant difference overall among modafinil groups and placebo ( $\mathrm{p}=0.63$, overall Kruskal-Wallis). The largest betweengroups difference on this measure was for Modafinil 200mg vs. placebo, 13.8 days vs. 11.2 days. For the secondary outcome of terminal abstinence, we again found no significant difference overall among treatment groups (Fisher's Exact $\mathrm{p}=0.84$ ). 'Successes' occurred as follows: placebo: $12 / 68$, modafinil 200mg: 10/72, and modafinil 400mg: $11 / 70$, or a total of $33 / 210$ participants, nearly $16 \%$. The difference between placebo and combined modafinil groups was also not significant $(\mathrm{p}=0.69)$

\subsubsection{Efficacy in medication-compliant participants, modafinil groups only-} Our ad-hoc study of modafinil compliance did find a significant difference in longest duration of abstinence or 'maximum number of consecutive methamphetamine non-use days' ( 23 days vs. 10 days, $\mathrm{p}=0.003$ ), between those having the top quartile of compliance (>85\% of weekly urines were modafinil positive, $\mathrm{N}=36$ ), and the rest of those who were in modafinil treatment groups $(\mathrm{N}=106)$. Modafinil compliance was also associated with better retention ( $\mathrm{p}=0.0014, \log$-rank, Fig. 5).

\subsection{Attention Deficit Hyperactivity Disorders}

None of the comparisons of change scores for the Adult ADHD Investigator Symptom Rating Scale was significantly different. The largest observed difference was between placebo and modafinil 200mg from baseline to Week 8, a greater decline (improvement) for modafinil of almost 3 points on a scale of $0-54$ possible points $(-2.7, p=0.09$, KruskalWallis). The proportion of participants who improved (decreased their score) by a threshold of 30\% or more on the AISRS was not significantly different among treatment groups. Those who improved $30 \%$ represented $40 \%$ of the total who had a change score from 
baseline to last AISRS, 66/165. Similarly, the Barratt's Impulsiveness Scale did not show significant treatment group differences by ANOVA p-values at baseline, Week 6 or Week 12 , in either the total scale or the. Dividing the total sample into 'normal' vs. 'high' impulsivity groups ( $<72$ vs. $\geq 72$ score, BIS) showed no significant difference in non-use weeks by impulsivity.

The CANTAB tests showed that one cognitive measure, Pattern Recognition Memory, had a nominally statistically significant beneficial effect with modafinil treatment. The immediate recognition part of the test showed improved accuracy among 'completers' $(\mathrm{N}=112)$ with the 200mg dose at Week $6(\mathrm{p}=0.044$, ANOVA), but not at Week 12. The 20-minute-delayed recognition task showed improved accuracy with 400mg at Week 12 ( $\mathrm{p}=0.049$, ANOVA) and marginally at 'last available score'. Another measure, Rapid Visual Information Processing, showed a possible detrimental effect of modafinil: the $400 \mathrm{mg}$ dose had increased false alarms at Week 12. No other significant effects were noted from the tests in the assessment.

\subsection{Other Secondary Outcomes}

We found no significant differences overall between modafinil and placebo treatment groups in all of our secondary outcome measures, including: 1) for quantitative methamphetamine use, ( $\mathrm{p}=0.43, \mathrm{GEE}$ ); 2) Addiction Severity Index (ASI)-Lite domains, (all $\mathrm{p}>0.118$, Kruskal-Wallis); 3) Clinical Global Impression scales, for the Observer (CGI-O, $p>0.45$ ) or the Self (CGI-S, $p>0.13$, GEE); 4) BSCS total score ( $p=0.47$, GEE); and 5) the HIV Risk Behavior Scale (HRBS) sub-scores or the overall ( $\mathrm{p}=0.13,0.44$, and $0.16, \mathrm{~K}-\mathrm{W})$.

\subsection{Safety}

There were four Serious Adverse Events (SAEs) that occurred during the treatment or follow-up phase of the study, three events with modafinil $400 \mathrm{mg}$ and one event with modafinil 200mg. These were: suicidal ideation with multiple drug overdose; chest pain (not myocardial infarction); ischemic injury / compartment syndrome of the forearm; and cellulitis of the inner thigh / methicillin resistant Staph aureus. These were considered not related to the study agent.

Our non-serious Adverse Events totaled 1674 events among 182 participants. Among the events that occurred more frequently with modafinil than with placebo, and occurred in participants at a $>5 \%$ rate, were cough (17\%), insomnia (16\%), nausea (15\%), dizziness (11\%), anxiety (10\%), depression (8\%), arthralgia (8\%), dry mouth (6\%), dyspepsia (6\%), neck pain (5\%), as well as the total events from these Body Systems: Psychiatric Disorders ( $35 \%, \mathbf{p}=\mathbf{0 . 0 5})$, Musculoskeletal $(31 \%, \mathrm{p}=0.36)$, and Investigations [body temperature increased, heart rate inc., $\gamma$-glutamyl-transferase inc., etc.] ( $7 \%, \mathbf{p}=\mathbf{0 . 0 5})$.

There were four participants who discontinued the study drug and still completed the study, two of whom were on placebo. The other two who stopped drug and still completed the study were both taking modafinil 400mg. One participant took study drug on Day 1 and 2, then discontinued "due to anxiety." This participant tried again the first day of Week 4, then discontinued permanently "due to anxiety." Another participant took modafinil 400mg for two days then stopped permanently "due to rash."

\section{Discussion}

In this study, the effect of modafinil on the planned primary outcome (methamphetamine non-use weeks) was not significant. Also, the 'terminal abstinence' outcome, which is recently preferred by the FDA and Cochrane reviews, did not show a medication effect. 
In addition, other secondary outcome measures did not show significant effects of modafinil to decrease the amount of meth used or increase the length of abstinence (prevent relapse). However, the measurement of study drug in the urine, from modafinil groups only, showed that only about $50 \%$ of participants were 'compliant' with medication, while also demonstrating better retention and a longer maximum duration of abstinence in the top quartile of participants who took most of their study drug. Irrespective of treatment group, there was a significant increase in methamphetamine-free weeks over time, which indicates that something about the behavioral treatment or being in the study, aside from modafinil, was associated with decreased meth use.

Our post-hoc finding in the 'good compliance' group is not good evidence of a causal relationship between taking modafinil and the improved outcome, because the comparison did not include the placebo group. The longer abstinence from meth (23 days) observed in the top quartile of compliance could be caused, for example, by the longer stay in treatment or by some other unmeasured factor, which is also associated with taking more modafinil. There could be many explanations for the apparent associations, such as external effects on the price or availability of meth, or genetic factors that are not related to modafinil. To control for the possible confounding effects, a subsequent efficacy study should make observations of compliance in both the active and placebo groups, so as to preserve the virtues of randomization in this difficult population.

Such a compliance study would raise related questions, such as: how much compliance with the active treatment should be considered 'enough'? In our study, we arbitrarily chose the top quartile of urine modafinil concentrations so as to have an exemplary group to compare to the rest. This 'best compliance' group happened to have at least a measurable amount of modafinil in at least $85 \%$ of weekly urine specimens. Previous informal discussions with the FDA have suggested that an estimated $75 \%$ compliance may be an adequate uptake of treatment, but it seems quite possible that lower levels of compliance with an effective medication could show a beneficial effect. If another study started with compliance data from all treatment groups, the analysis could look at several 'threshold' levels, or at a continuous relationship between the observed amount of medication in the body and its effect on meth use (as with a dose-response curve). It will also be important to determine whether active and placebo groups comply with taking study drug at the same or substantially different rates.

Regarding symptoms of ADHD present in our population, $21 \%$ of participants dropped out before their Week 8 re-assessment. Of those who provided a change score, $40 \%$ had a meaningful improvement, but apparently not related to modafinil. The cognitive tests change scores also showed only weak association with modafinil, which might be expected with the poor medication compliance that was demonstrated.

Regarding generalizability, the exclusion of those who had any lifetime history of alcohol dependence may make our sample somewhat different from the larger population that has meth dependence. Among the 440 who were screened for this trial, $11 \%$ failed because of dependence on another psychoactive substance, which included alcohol, opiates or sedatives.

Our adverse events were, in general, just what we expected from the FDA-approved label for this drug. We listed "side effects" from the label in our Informed Consent Form, including "headache, nausea, nervousness, anxiety," as well as "rare reports of serious lifethreatening skin reactions," which later appeared in some form in our collection of adverse events. The FDA label for modafinil notes a dose-dependence for two kinds of adverse event - headache and anxiety (Cephalon, Inc., 2007). We noted discontinuation of the study 
drug with anxiety and rash. In our study, the 400mg dose of modafinil may have been associated with more frequent discontinuation of the medication ( 2 events vs. 0 and 2).

Our results are similar to the negative findings of other recent studies of modafinil for methamphetamine dependence (Shearer et al., 2009; Heinzerling et al., 2010). The fact that only $16 \%$ of all our participants remained in treatment until the end of 12 weeks, to achieve a relatively meager 2 -week abstinence, shows that stimulant addiction remains a very difficult illness, for which an effective medication would be of great benefit.

\section{Acknowledgments}

Role of Funding Source: Funding for this study was provided by NIDA Contract\# Y1-DA-4006-01. The National Institute on Drug Abuse-NIH had a major role in study design, in the analysis and interpretation of data, and in the writing and submission of this manuscript for publication.

\section{References}

Adler LA, Spencer T, Faraone SV, Reimherr FW, Kelsey D, Michelson D, Biederman J. Training raters to assess adult ADHD: reliability of ratings. J Atten Disord. 2005; 8:121-126. [PubMed: 16009660]

Anderson AL, Reid MS, Li SH, Holmes T, Shemanski L, Slee A, Smith EV, Kahn R, Chiang N, Vocci F, Ciraulo D, Dackis C, Roache JD, Salloum IM, Somoza E, Urschel HC 3rd, Elkashef AM. Modafinil for the treatment of cocaine dependence. Drug Alcohol Depend. 2009; 104:133-139. [PubMed: 19560290]

Ballon JS, Feifel D. A systematic review of modafinil: potential clinical uses and mechanisms of action. J Clin Psychiatry. 2006; 67:554-566. [PubMed: 16669720]

Cephalon, Inc.. Cephalon, Inc.; Frazer, PA: 2007. PROVIGIL® (modafinil) Tablets [C-IV], FDA Approved Labeling - dated August 17, 2007. www.accessdata.fda.gov/drugsatfda_docs/label/2007/020717s020s013s018lbl.pdf

Dackis CA, Kampman KM, Lynch KG, Pettinati HM, O'Brien CP. A double-blind, placebo-controlled trial of modafinil for cocaine dependence. Neuropsychopharmacology. 2005; 30:205-211. [PubMed: 15525998]

Drug Enforcement Administration. Washington, D.C. 20537: 2005. Guidelines for Law Enforcement for the Cleanup of Clandestine Drug Laboratories - 2005 Edition. http://www.justice.gov/dea/resources/redbook.html

Elkashef AM, Rawson RA, Anderson AL, Li SH, Holmes T, Smith EV, Chiang N, Kahn R, Vocci F, Ling W, Pearce VJ, McCann M, Campbell J, Gorodetzky C, Haning W, Carlton B, Mawhinney J, Weis D. Bupropion for the treatment of methamphetamine dependence. Neuropsychopharmacology. 2008; 33:1162-1170. [PubMed: 17581531]

Ferraro L, Antonelli T, Tanganelli S, O'Connor WT, Perez de la Mora M, Mendez-Franco J, Rambert FA, Fuxe K. The vigilance promoting drug modafinil increases extracellular glutamate levels in the medial preoptic area and the posterior hypothalamus of the conscious rat: prevention by local GABA-A receptor blockade. Neuropsychopharmacology. 1999; 20:346-356. [PubMed: 10088135]

Glasner-Edwards S, Mooney LJ, Marinelli-Casey P, Hillhouse M, Ang A, Rawson RA. Methamphetamine Treatment Project Corporate Authors. Psychopathology in methamphetaminedependent adults 3 years after treatment. Drug Alcohol Rev. 2010; 29:12-20. [PubMed: 20078677]

Grinspoon, L.; Hedblom, P. The Speed Culture - Amphetamine Use and Abuse in America. Harvard University Press; Cambridge, MA: 1975.

Heinzerling KG, Swanson AN, Kim S, Cederblom L, Moe A, Ling W, Shoptaw S. Randomized, double-blind, placebo-controlled trial of modafinil for the treatment of methamphetamine dependence. Drug Alcohol Depend. 2010; 109:20-29. [PubMed: 20092966]

Hser YI, Evans E, Huang D, Brecht ML, Li L. Comparing the dynamic course of heroin, cocaine, and methamphetamine use over 10 years. Addict Behav. 2008; 33:1581-1589. [PubMed: 18790574]

Jasinski DR. An evaluation of the abuse potential of modafinil using methylphenidate as a reference. J Psychopharmacol. 2000; 14:53-60. [PubMed: 10757254] 
Lindsay SE, Gudelsky GA, Heaton PC. Use of modafinil for the treatment of attention deficit/ hyperactivity disorder. Ann Pharmacother. 2006; 40:1829-1833. [PubMed: 16954326]

McGregor C, Srisurapanont M, Mitchell A, Wickes W, White JM. Symptoms and sleep patterns during inpatient treatment of methamphetamine withdrawal: a comparison of mirtazapine and modafinil with treatment as usual. J Subst Abuse Treat. 2008; 35:334-342. [PubMed: 18329221]

Myrick H, Malcolm R, Taylor B, LaRowe S. Modafinil: preclinical, clinical, and post-marketing surveillance-a review of abuse liability issues. Ann Clin Psychiatry. 2004; 16:101-109. [PubMed: 15328903]

Nguyen D, Smith LM, Lagasse LL, Derauf C, Grant P, Shah R, Arria A, Huestis MA, Haning W, Strauss A, Della Grotta S, Liu J, Lester BM. Intrauterine growth of infants exposed to prenatal methamphetamine: results from the infant development, environment, and lifestyle study. $\mathrm{J}$ Pediatr. 2010; 157:337-339. [PubMed: 20570284]

Reichel CM, See RE. Modafinil effects on reinstatement of methamphetamine seeking in a rat model of relapse. Psychopharmacology. 2010; 210:337-346. [PubMed: 20352413]

Scammell TE, Estabrooke IV, McCarthy MT, Chemelli RM, Yanagisawa M, Miller MS, Saper CB. Hypothalamic arousal regions are activated during modafinil-induced wakefulness. J Neurosci. 2000; 20:8620-8628. [PubMed: 11069971]

Shearer J, Darke S, Rodgers C, Slade T, van Beek I, Lewis J, Brady D, McKetin R, Mattick RP, Wodak A. A double-blind, placebo-controlled trial of modafinil (200 mg/day) for methamphetamine dependence. Addiction. 2009; 104:224-233. [PubMed: 19149817]

Stone EA, Cotecchia S, Lin Y, Quartermain D. Role of brain alpha 1B-adrenoceptors in modafinilinduced behavioral activity. Synapse. 2002; 46:269-270. [PubMed: 12373742]

Substance Abuse and Mental Health Services Administration. Office of Applied Studies, NSDUH Series H-38B, HHS Publication No SMA 10-4856 Appendices. Rockville, MD: 2010. Results from the 2009 National Survey on Drug Use and Health: Volume II Technical Appendices and Selected Prevalence Tables. http://oas.samhsa.gov/nsduhLatest.htm

Taneja I, Haman K, Shelton RC, Robertson D. A randomized, double-blind, crossover trial of modafinil on mood. J Clin Psychopharmacol. 2007; 27:76-79. [PubMed: 17224718]

Turner DC, Clark L, Dowson J, Robbins TW, Sahakian BJ. Modafinil improves cognition and response inhibition in adult attention-deficit/hyperactivity disorder. Biol Psychiatry. 2004; 55:1031-1040. [PubMed: 15121488]

United Nations Office on Drugs and Crime. World Drug Report 2010. United Nations Publications; New York, NY: 2010. http://www.unodc.org/unodc/en/data-and-analysis/WDR-2010.html

Wei LJ, Lachin JM. Properties of the urn randomization in clinical trials. Control Clin Trials. 1988; 9:345-364. [PubMed: 3203525]

Wisor JP, Nishino S, Sora I, Uhl GH, Mignot E, Edgar DM. Dopaminergic role in stimulant-induced wakefulness. J Neurosci. 2001; 21:1787-1794. [PubMed: 11222668] 


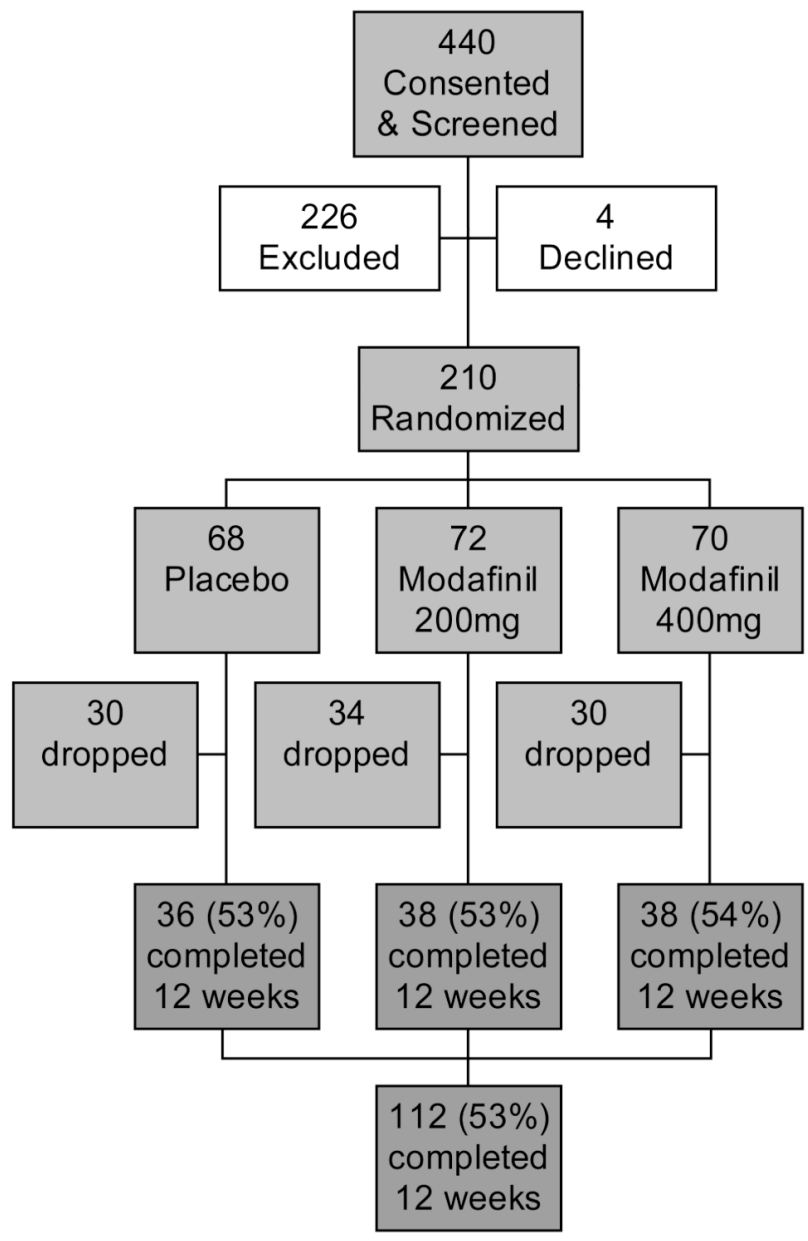

1.

Flow chart for numbers of participants screened, randomized, and treated in a clinical trial of modafinil for methamphetamine dependence. 


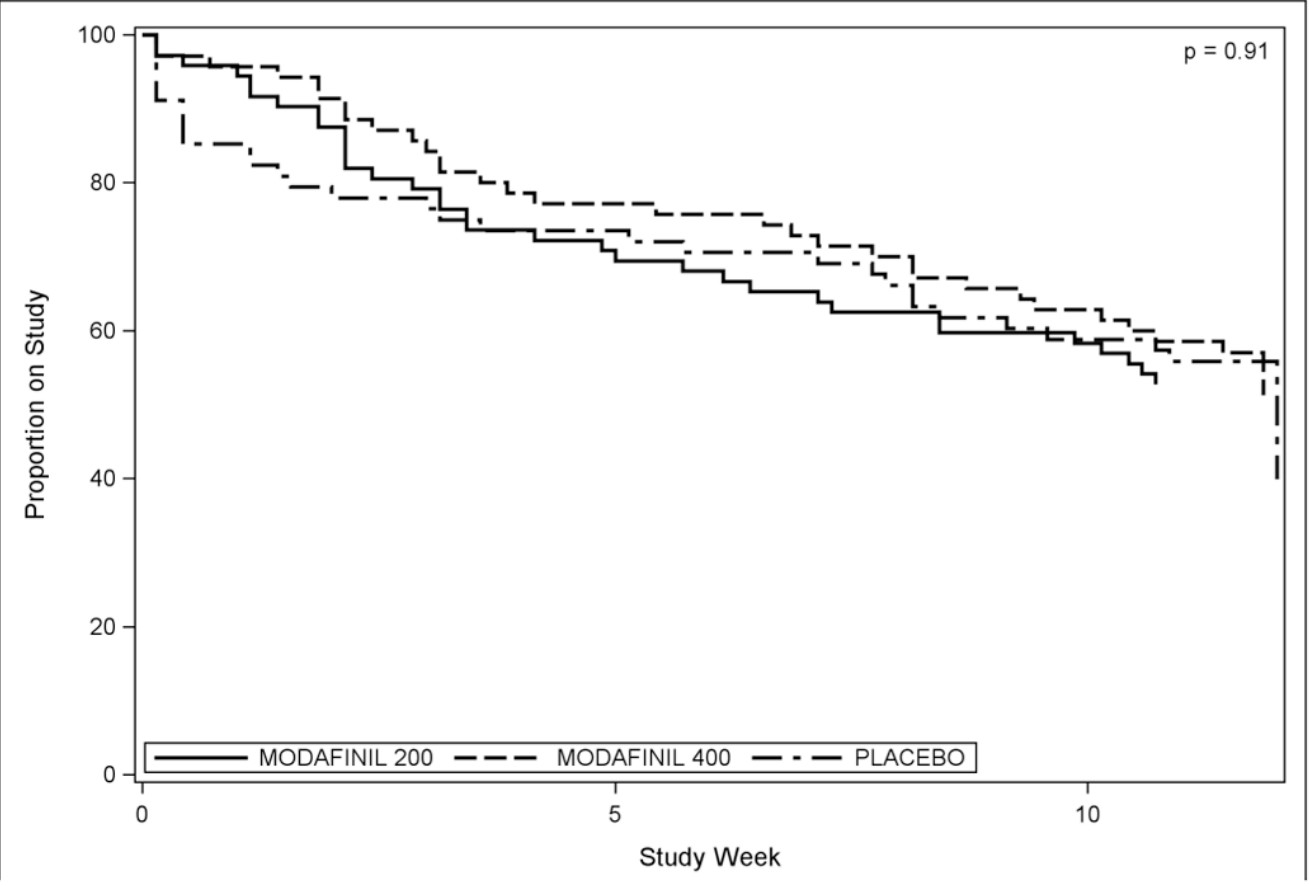

2.

Retention, or percent of participants remaining in the study over 12 weeks, by treatment group. 


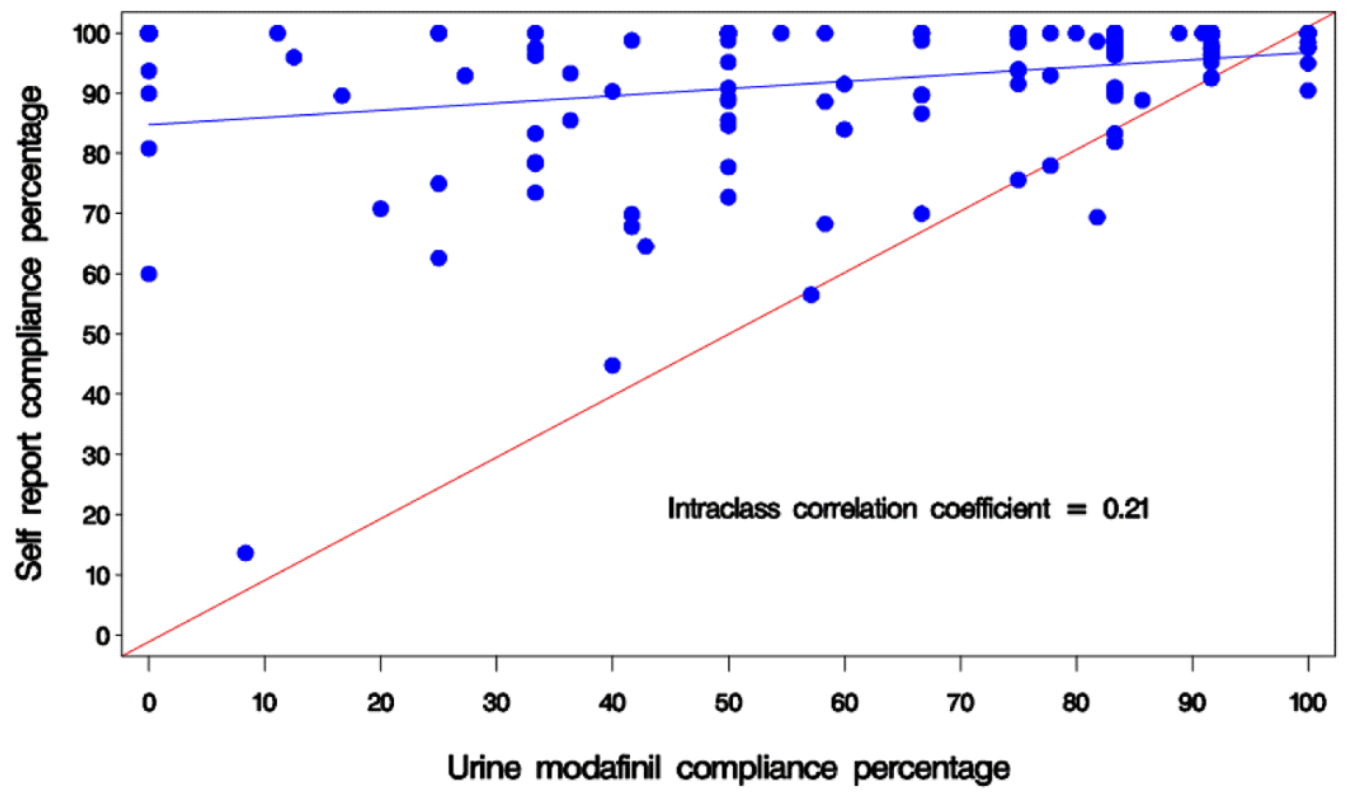

3.

Compliance correlation, or percent of expected modafinil taken, self-report (aided by pill count) vs. weekly urine assay. 


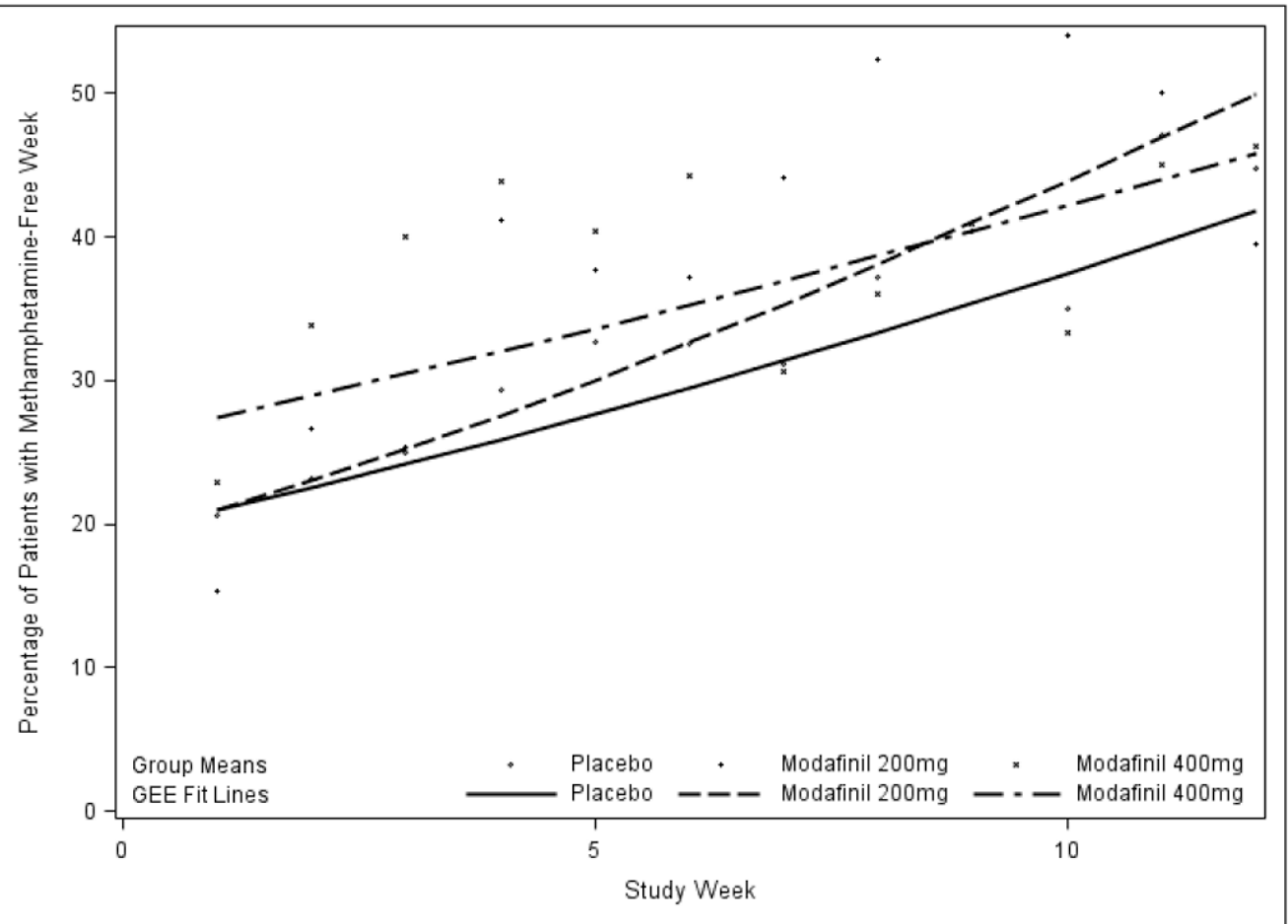

4.

Efficacy, or percent of participants having a methamphetamine non-use week (all urines negative) over 12 weeks, by treatment group. GEE fit lines and group means. 


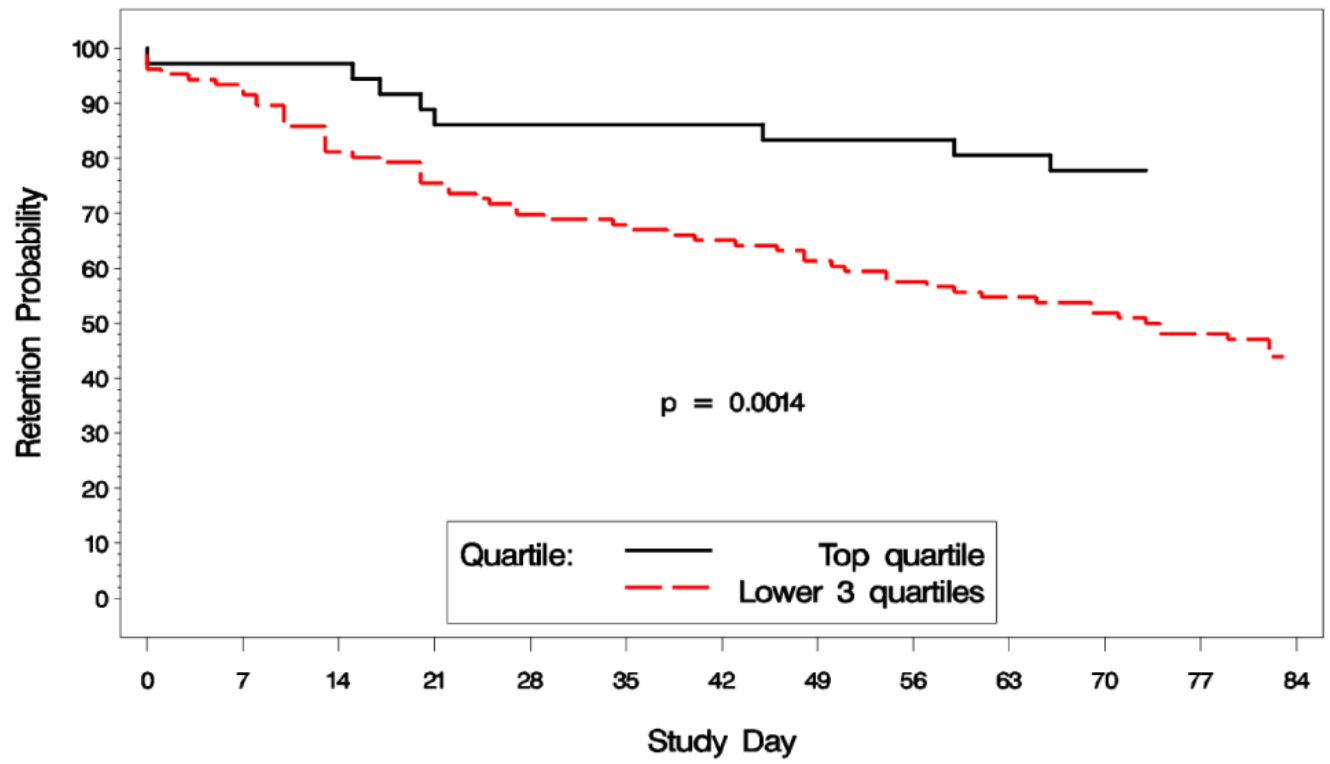

5.

Retention by modafinil compliance groups, top quartile of compliance vs. remaining three quartiles, percent of participants remaining in the study over 12 weeks. 


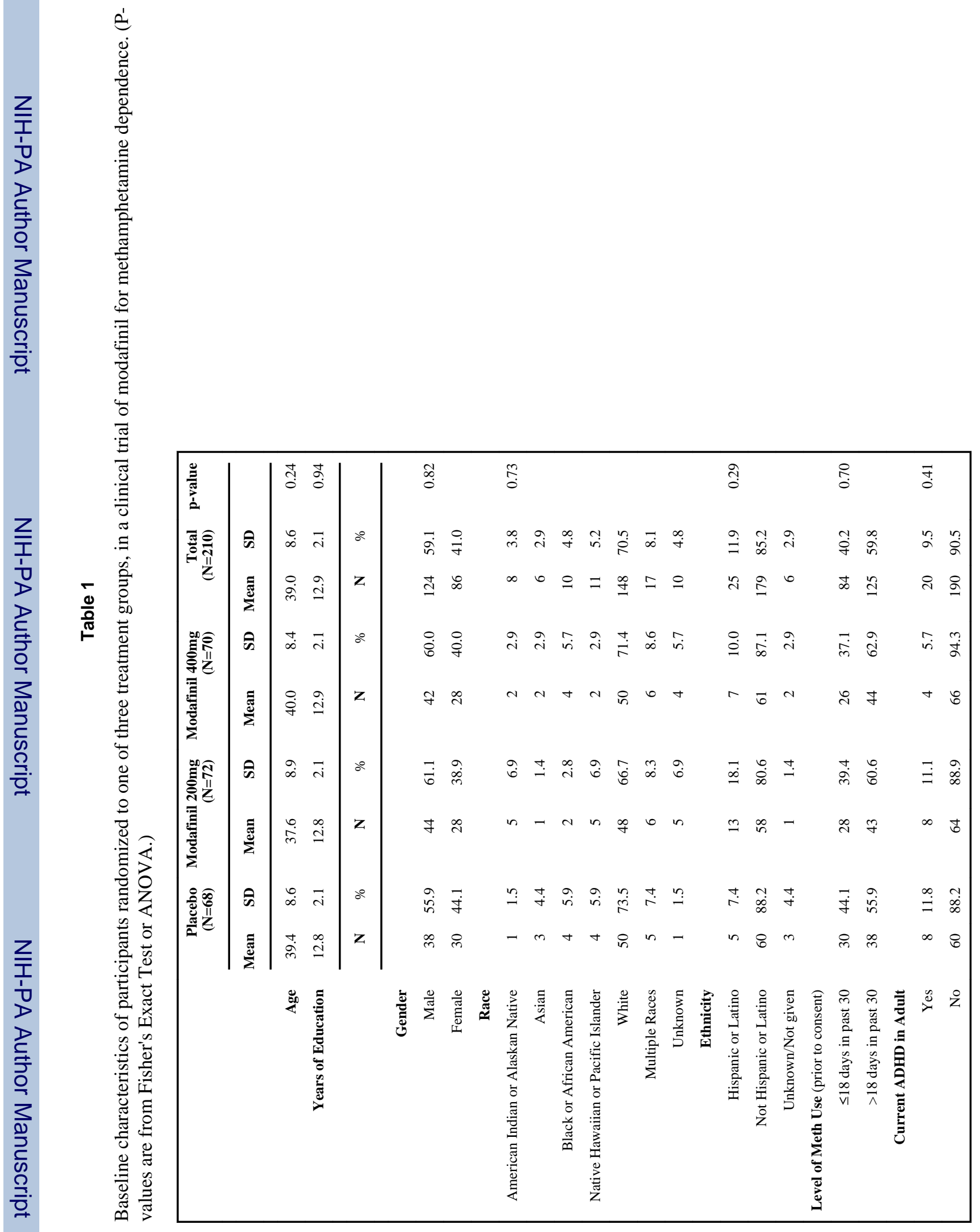

Drug Alcohol Depend. Author manuscript; available in PMC 2013 January 1. 